\title{
Inpatient satisfaction with medical information received from caregivers: an observational study on the effect of social deprivation
}

\author{
L. Moret ${ }^{1,2^{*}}$ (D, E. Anthoine ${ }^{1,2}$, A. Pourreau ${ }^{1}$, F. Beaudeau ${ }^{3}$ and B. Leclère ${ }^{1}$
}

\begin{abstract}
Background: The main objective of this study was to explore the relationships between inpatients' social differentiation and satisfaction with the medical information delivered by caregivers.

Methods: In four departments of a teaching hospital, patients were enrolled as well as their attending physician and one of the nurses assigned to them. Structured survey questionnaires were administered face-to-face to patients and caregivers. Patients were asked to rate their satisfaction with the medical information received, the quality and duration of the interactions with the caregivers, and their experience regarding their involvement in medical decision-making. Caregivers were asked to rate their perception of the patients' social position and involvement in medical decision-making. Social deprivation was assessed using the EPICES score in particular. The statistical analysis was mainly descriptive and completed by a structural equation model.

Results: A sample of 255 patients, 221 pairs of patient-physician and 235 pairs of patient-nurse were considered. One third of the patients (32.7\%) were identified as socially deprived. They were significantly less satisfied with the information they received on their health status or their treatment; $56.7 \%$ of patients thought that they received sufficient explanations without having to ask. This proportion was significantly lower in socially deprived patients (42.3\%) compared to not deprived patients $(63.6 \%, p<0.01)$. Patients' reported involvement in medical decisionmaking was significantly lower for socially deprived patients (75.0\% vs $89.0 \%, p<0.001)$. The structural equation model showed that the main determinant of patients' satisfaction regarding medical information was their perceived involvement in informed medical decision-making (CFI = 0.998, RMSEA =0.022).
\end{abstract}

Conclusions: These findings suggest that physicians and nurses need training on communication targeted towards vulnerable patients, in order to improve the accessibility of medical information, and thus to reduce health inequalities.

Keywords: Inpatient satisfaction, Medical information, Shared decision-making, Social deprivation

\footnotetext{
* Correspondence: Imoret@chu-nantes.fr

${ }^{1}$ Public Health Department, University Hospital, Saint-Jacques Hospital, 85 rue Saint-Jacques, 44093 Nantes Cedex, France

2UMR 1246 INSERM SPHERE "MethodS in Patients-centered outcomes and

HEalth ResEarch", University of Nantes, Bd Benoni-Goullin, 44200 Nantes,

France

Full list of author information is available at the end of the article
}

(c) The Author(s). 2017 Open Access This article is distributed under the terms of the Creative Commons Attribution 4.0 International License (http://creativecommons.org/licenses/by/4.0/), which permits unrestricted use, distribution, and reproduction in any medium, provided you give appropriate credit to the original author(s) and the source, provide a link to the Creative Commons license, and indicate if changes were made. The Creative Commons Public Domain Dedication waiver (http://creativecommons.org/publicdomain/zero/1.0/) applies to the data made available in this article, unless otherwise stated. 


\section{Background}

Patient information is one of the key elements of the physician-patient relationship [1-4]. It has been shown to be strongly linked to patients' satisfaction and compliance, but also to morbidity measures [5, 6]. Among the existing models of interaction, the traditional asymmetric physicianpatient relationship-the dominant "paternalistic model"-is slowly being replaced by more patient-centred models, based on negotiation and cooperation. For example, Street et al. highlighted the importance of a shared identity between patient and physician, combining reciprocity and mutual influence $[3,7]$. It has also been shown that patients taking an active role during physician-patient interactions were more inclined to ask questions, understand medical explanations and make good decisions about their care $[8,9]$.

However, the physician-patient relationship still usually remains unequal, which can induce health inequalities. First, physicians' beliefs about their patients can lead to disparities in treatment [10]. Second, the interactions between physicians and patients seem to vary according to their perceived social distance [11-14]. Several studies showed that patients from lower social classes receive significantly less information and are significantly less involved in shared decision-making [15-18]. Indeed, these patients are usually less proactive in eliciting information and their physicians frequently underestimate their need for it. For example, it has been shown that physicians are less likely to discuss cancer screening tests or post-mastectomy reconstruction with patients who had a lower education level $[16,18]$. In the United States, where ethnicity and socioeconomic status are strongly correlated, African-American patients seem to receive less information from their physician after an angiography [15] or in the context of kidney transplantation [19].

Most of the published studies have explored these interactions during medical encounters, but we hypothesize that this inequality in patients' information also occurs in other contexts, especially within hospitals, where patients usually interact with several caregivers and not solely their attending physician. In this context, disagreements between health care professionals concerning their respective roles in information delivery may also occur. In a study conducted by our team, nurses considered that providing patients with information was an integral part of their mission (giving explanations during care provision, relaying, re-explaining and completing the information given by the physicians). However, nurses also reported that they usually did not know what the patients were told by the physicians, which complicated their task and hindered the quality of the information delivered. Physicians, in contrast, had a much more restrictive view of patient information, only considering it during a face-to-face interview [20].
The main objective of this study was to explore the relationship between inpatients' social differentiation and their satisfaction with the medical information delivered by the caregivers. The secondary objectives were to explore the relationship of inpatients' social differentiation with their satisfaction regarding their interactions with the caregivers, and with their experience regarding their involvement in shared decision-making.

\section{Methods}

\section{Ethical and consent considerations}

The research protocol was approved by the "Groupe nantais d'éthique dans le domaine de la santé" (Nantes ethics committee in health research). All eligible inpatients, physicians and nurses were invited to participate by the interviewer and gave their verbal informed consent before the interview. The data were anonymized before recording. According to the L1121-1 and R1121-2 articles of the French code of public health, written consent and IRB approval are not necessary for noninterventional research.

\section{Study population and setting}

This study was conducted during the second semester of 2011 at the Nantes University Hospital (France). It involved 10 voluntary units from two surgery departments (gynaecology and orthopaedic surgery) and two medicine departments (internal medicine and emergency medicine). Inpatients were recruited $24 \mathrm{~h}$ before discharge. To ensure the inclusion of enough socially deprived patients, that means patients who cannot have an easy and frequent access to the many different aspects of their culture and society, due to a combination of factors such as low socioeconomic status or poor education, the minimum sample size to be considered was 172 , under the assumption that socially deprived patients represented $35 \%$ of the whole population of inpatients [21]. A total of 333 patients fulfilled the eligibility criteria for inclusion (age $\geq 18$, proficiency in French, hospitalization $\geq 3$ days, informed consent given), but 47 of them left the hospital before being interviewed, and 31 finally refused to participate. Therefore, 255 patients actually participated (participation rate: $76.6 \%$ ). To ensure a high response rate, patient questionnaires were completed by trained interviewers during a face-to-face interview of 15 to $30 \mathrm{~min}$.

For each patient, the attending physician and one of the assigned nurses were also recruited. Once the patients were interviewed, their attending physician and nurse were contacted and interviewed face-to-face, on the day of discharge or within the next 2 days. The interviews lasted from $2 \mathrm{~min}$ for nurses to $5 \mathrm{~min}$ for physicians. Every caregiver accepted to participate, but some of them were not available at the time of interview 
and some patients did not have a designated attending physician. The final sample consisted of 221 patientphysician and 235 patient-nurse pairs (i.e. respectively 86.7 and $92.2 \%$ of the patient sample).

\section{Measures \\ Patient questionnaire}

Patients' status towards social deprivation The patients' deprivation status was assessed in two ways by the patient himself or herself: an objective measure using a standardised score and a self-perceived measure. The individual index used to objectively measure the level of deprivation was the EPICES score ("Evaluation de la Précarité et des Inégalités de santé dans les Centres d'Examens de Santé" - Evaluation of Deprivation and Inequalities in Health Examination Centers). This score was validated in 2002 on a cohort of 197,389 subjects examined in 58 French health examination centers [21, 22]. It consists of 11 items related to isolation (one item), health insurance status (one item), economic status (three items), social support (three items) and leisure activity (three items) (Table 1). For each item, a binary response Yes/No is expected from the patient. The total EPICES scores range from 0 to 100 , from the lowest to the highest social deprivation. Even though this score can be considered as continuous, it has been mostly used with a threshold to categorize patients' deprivation status [22-24]: patients were considered deprived if their score is superior to 30.17 (which correspond to the fourth quintile of the score distribution in the original study). In Table 2, deprived patients were identified using this threshold.

Table 1 Description of the 11 EPICES items scale. For each item, a binary response Yes/No is expected from the patient

1. Do you sometimes meet with a social worker (welfare worker, educator)?

2. Do you have complementary health insurance (mutual insurance)?

3. Do you live as a couple?

4. Are you a homeowner or will you be one in the near future?

5. Are there periods in the month when you have real financial difficulties in facing you needs (food, rent, electricity)?

6. Have you participated in any sports activities in the last 12 months?

7. Have you gone to any shows (cinema, theatre) in the last 12 months?

8. Have you gone on holiday during the past 12 months?

9. Have you seen any family members in the past 6 months (other than your parents or children)?

10. Did you have difficulties (financial, family or health), is there anyone around you who could take you in for a few days?

11. Did you have difficulties (financial, family or health), is there anyone around you who could help you financially (material aid such as lending you money)?
Table 2 Main patient's characteristics $(n=255)$

\begin{tabular}{|c|c|c|c|c|}
\hline \multirow{2}{*}{$\begin{array}{l}\text { Patients' } \\
\text { characteristics }\end{array}$} & \multicolumn{3}{|l|}{ Patients } & \multirow[t]{2}{*}{$p$} \\
\hline & $\begin{array}{l}\text { Whole } \\
\text { sample (\%) }\end{array}$ & Deprived $^{a}(\%)$ & $\begin{array}{l}\text { Not } \\
\text { deprived (\%) }\end{array}$ & \\
\hline \multicolumn{5}{|l|}{ Gender } \\
\hline Male & 34.5 & 34.6 & 33.5 & \multirow[t]{2}{*}{$N S^{* *}$} \\
\hline Female & 65.5 & 65.4 & 67.5 & \\
\hline \multicolumn{5}{|l|}{ Age group (years) } \\
\hline$\geq 65$ & 43.1 & 59.3 & 34.7 & \multirow[t]{2}{*}{$<0.01$} \\
\hline$<65$ & 56.9 & 40.7 & 65.3 & \\
\hline \multicolumn{5}{|l|}{ Educational level } \\
\hline High & 25.9 & 14.8 & 32.3 & \multirow[t]{3}{*}{$<0.01$} \\
\hline Medium & 38.4 & 35.8 & 39.5 & \\
\hline Low & 35.7 & 49.4 & 28.2 & \\
\hline \multicolumn{5}{|l|}{ Employment status } \\
\hline Employed active & 30.6 & 16.0 & 37.7 & \multirow[t]{3}{*}{$<0.01$} \\
\hline Retired & 50.4 & 60.5 & 45.5 & \\
\hline Other inactive & 19.0 & 23.5 & 16.8 & \\
\hline Having a referring GP & 97.6 & 95.1 & 99.4 & $<0.05$ \\
\hline \multicolumn{5}{|c|}{ Chronically diseased or handicapped } \\
\hline Yes & 65.9 & 71.6 & 63.4 & \multirow[t]{2}{*}{ NS } \\
\hline No & 34.1 & 28.4 & 36.6 & \\
\hline \multicolumn{5}{|c|}{ Perceived health status (compared to people of the same age) } \\
\hline Lower & 34.0 & 31.2 & 36.9 & \multirow[t]{2}{*}{ NS } \\
\hline Similar or better & 66.0 & 68.8 & 63.1 & \\
\hline
\end{tabular}

${ }^{*} N S$ non-significant $(p>0.05)$

aEPICES score $>30.17$ on a scale from 0 to 100

In addition to this objective measure, the patients were also asked to rate their perceived social status on a scale from 1 to 10 .

Patients' satisfaction about the quality of the medical information they received from caregivers Patients' levels of satisfaction with the medical information they received (about health status, treatment, investigations and discharge) and with the quality and duration of their interactions with the caregivers were measured using 5 answer modalities (very satisfied, satisfied, moderately satisfied, dissatisfied, extremely dissatisfied).

Patients' perceived experience concerning their involvement in informed medical decision-making The patient were also asked if they felt that they were involved in medical decision-making and if they received sufficient information without having to ask. Five answer modalities were available: always, almost always, often, sometimes and never.

Finally, the questionnaire included descriptive demographic and socioeconomic variables: age, gender, educational level (high: high-school graduation and higher; 
medium: middle-school graduation; low: no formal diploma), employment status (employed active, retired, other inactive), declaration of a referring general practitioner (yes/no), existence of a chronic disease or handicap (yes/no).

\section{Questionnaire administrated to caregivers}

Physicians and nurses were asked separately to answer a specific questionnaire about their perception of their patient's social position on a scale from 1 to 10 , and about their perception on how the patient was involved in the medical decision making through the same 5 modalities as patients: always, almost always, often, sometimes and never.

\section{Statistical analysis}

Traditional descriptive statistics (frequency, median, mean \pm standard deviation) were used to describe the sample. The characteristics of deprived and not deprived patients were then compared by Chi-squared tests for categorical variables, trend Chi-squared test for ordinal variables and by $\mathrm{T}$-tests or paired $\mathrm{T}$-tests for continuous variables. Patients and caregivers' perception were compared considering the patient-nurse and patient-physician pairs separately.

As many of the measured variables were supposedly correlated, we chose to model the relationships between them with a structural equation model. First, we studied all pairwise Spearman correlation coefficients $(\rho)$ of the variables deemed as relevant based on the results of the bivariate analyses. The most correlated variables were grouped into latent factors that were used as independent variables in the final model. Diagonally weighted least squares were used to estimate the model parameters, and the full weight matrix was used to compute robust standard errors. Model selection was based on the comparative fit index (CFI) and the root mean square error of approximation (RMSEA).

All the analyses were performed using R 3.0.2. and IBM SPSS Statistics 19. Structural equation modelling was performed in $\mathrm{R}$ using the lavaan package version $0.5-20$ [25].

\section{Results}

\section{Characteristics of the sample}

The characteristics of the patient sample are described in Table 2. Two-thirds of the patients were females; the mean age was 59.1 years $(S D=21.4$; median of 60$)$. Half of the patients were retired and nearly two thirds were chronically ill or handicapped. Most of them (93\%) designated French as their mother tongue.

The distribution of the EPICES score in the sample is displayed in Fig. 1. This score was available for 248 patients. Overall, almost a third of them $(n=81 ; 32.7 \%)$ were identified as socially deprived. This proportion varied from $22 \%$ in the surgery departments to $52 \%$ in the emergency medicine department. Table 1 shows the description of the 11 EPICES items scale.

Comparisons between deprived and not deprived patients are available in Table 2. Deprived patients were significantly older, had a lower educational level and were more often

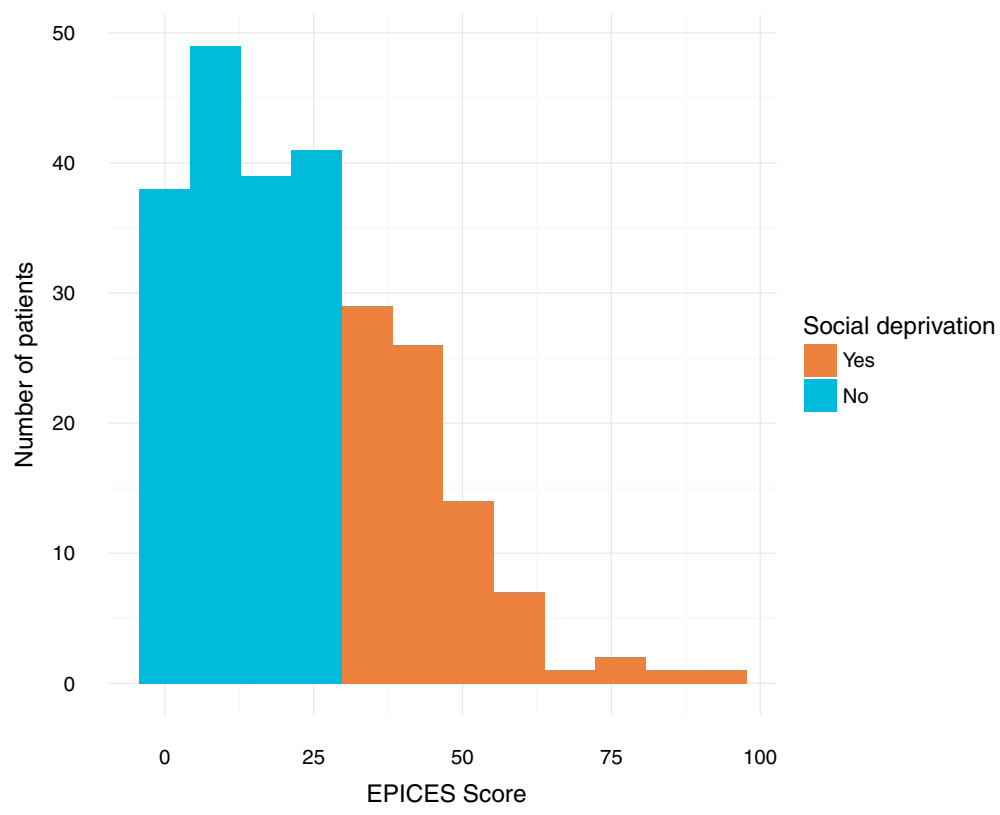

Fig. 1 Histogram of the EPICES score in the study sample $(n=248)$ 
retired than patients who were not socially deprived. In contrast, no significant differences were observed regarding chronic disease and handicap, and perceived health between the two groups.

\section{Comparisons between caregivers' and patients' perceptions of patient social level}

The average patients' self-perceived social level was 6.1 (SD = 1.5) out of 10 for the whole sample. Deprived patients gave on average significantly lower ratings than not deprived patients $(5.7(\mathrm{SD}=1.7)$ vs. 6.3 $(\mathrm{SD}=1.4) ; p<0.01)$. For the whole sample, the patients' perceived social level, as rated by the physicians and the nurses, averaged $6.5(\mathrm{SD}=2.0)$ and $6.5(\mathrm{SD}=1.6)$ out of 10 respectively. On average, the caregivers' ratings were significantly higher than the patients' self-ratings $(p<0.001)$, with differences of +1.11 for the physicians and +1.37 for the nurses. Physicians and nurses gave significantly $(p<0.001)$ lower ratings to deprived patients $(5.3(\mathrm{SD}=2.3)$ and $5.9(\mathrm{SD}=1.8)$ respectively) than to not deprived patients $(7.0 \quad(\mathrm{SD}=1.6)$ and $6.8(\mathrm{SD}=1.4)$ respectively).

\section{Patients' satisfaction about the quality of the medical information they received from caregivers}

More than $85 \%$ of the patients were satisfied with the medical information given by the caregivers (Fig. 2). Socially deprived patients were significantly less satisfied with the information they received on their health status or their treatment $(p<0.05)$. The quality of their care was perceived as excellent or very good by $61.4 \%$ of the patients, regardless they were deprived or not deprived (trend $p$-value: 0.57 ).

\section{Patients' perceived involvement in medical decision making}

Overall, only $56.7 \%$ of patients thought that they received enough explanations without having to ask for them. These results were significantly lower for socially deprived patients $(42.3 \%$ vs. $63.6 \%, p<0.01)$ (Fig. 3). More than $80 \%$ of patients perceived that they had always, almost always or often been involved in medical decision-making. This proportion was significantly lower for socially deprived patients $(75.0 \%$ vs. $89.0 \%, p<0.001)$ (Fig. 3).

In comparison, the proportion of patients having always or almost always been involved in medical-decision making was estimated at $83.7 \%$ by nurses and at $88.3 \%$ by physicians, with no significant difference between deprived and not deprived patients (trend $p$-values: 0.08 for nurses, 0.34 for physicians).

\section{Structural equation modelling}

The study of the Spearman's coefficients showed 1) strong correlations between the duration and the perceived quality of the interactions with caregivers $(\rho=0.86$ and 0.75 for nurses and physicians respectively); 2) a moderate correlation between the two variables relating to the perceived involvement of the patient in informed medical decision-making ("Did you receive explanations without having to ask for them?" and "Were you involved in medical decision

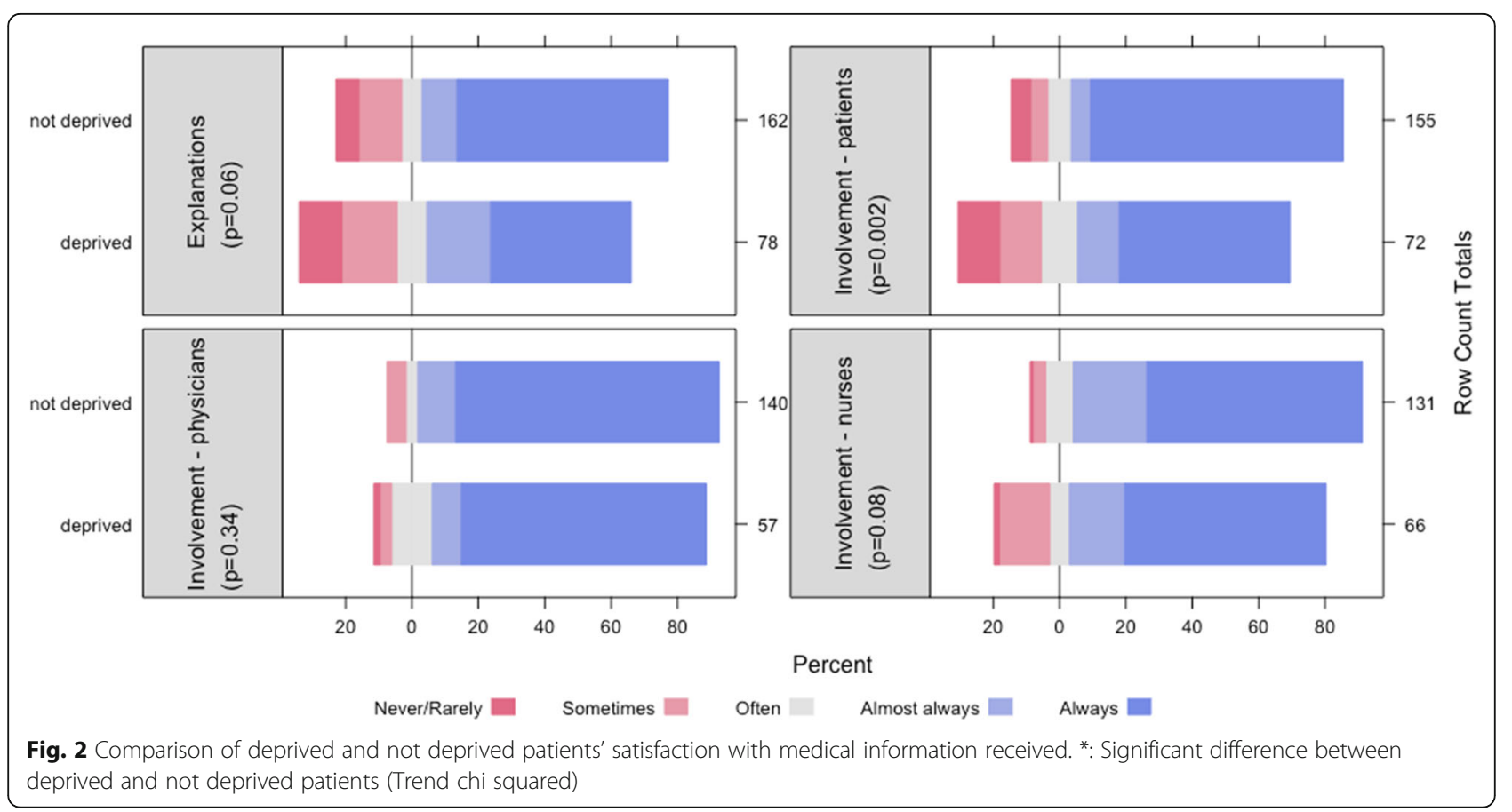




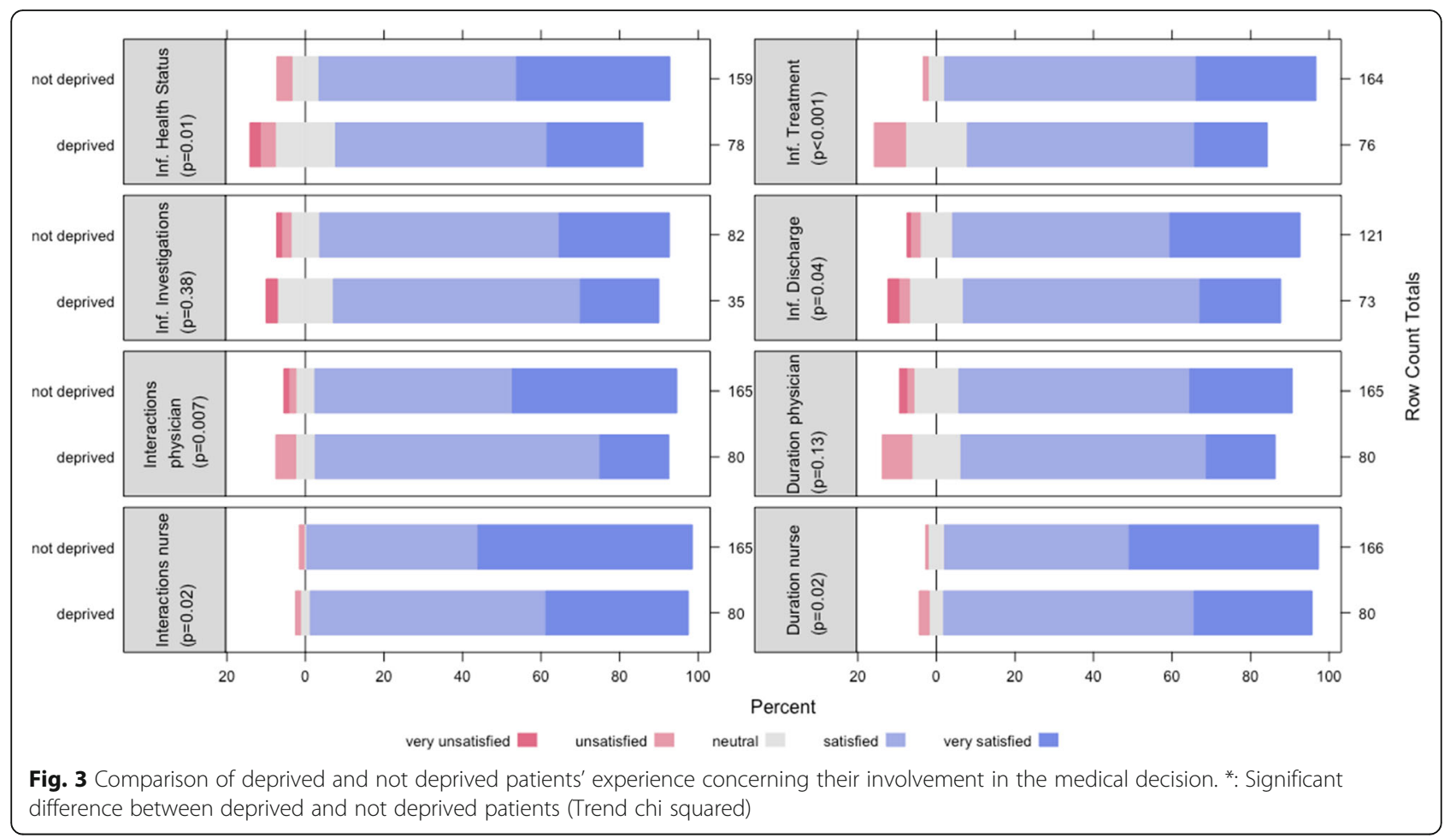

making?") $(\rho=0.67)$; and 3$)$ low to moderate correlations between two variables measuring the satisfaction of the information given regarding health status, treatments, investigations and discharge ( $\rho$ from 0.41 to 0.64 ). Four latent variables were created to reflect these correlations in the model ("relationship with nurse", "relationship with physician", "perceived involvement" and "satisfaction about information").

Figure 4 presents the final structural equation model and the estimated coefficients. The main determinant of patient satisfaction regarding medical information was the perceived involvement in informed medical decision-

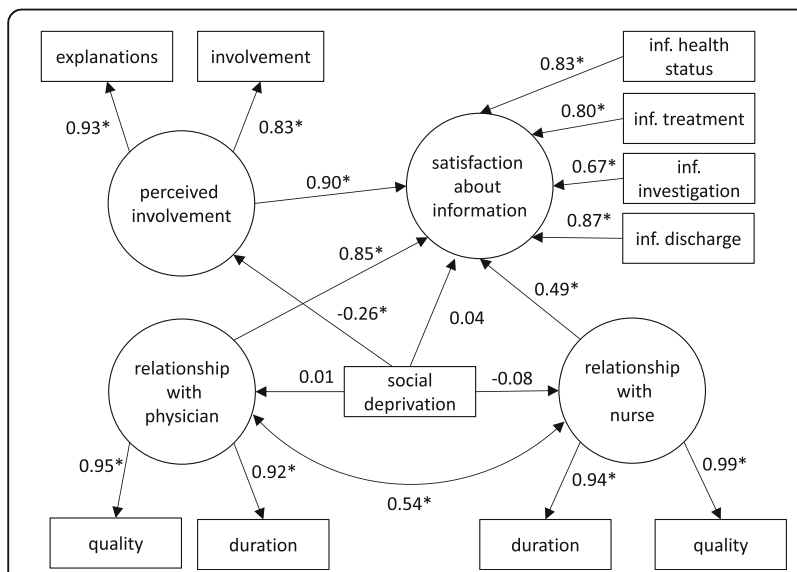

Fig. 4 Results of structural equation modelling. Numbers are standardized coefficients, the * indicates coefficients significantly different to zero making $(p<0.001)$. The qualities of the relationships with the physicians and, to a lesser extent, with the nurses also appeared as significant determinants (respectively $p=0.002$ and $p=0.001$ ). The direct effect of social deprivation on information satisfaction was not significant $(p=0.85)$, but a significant negative relationship between social deprivation and perceived involvement was observed $(p=0.001)$. Overall, this model displayed a very good fit to the data, with a CFI of 0.998 and a RMSEA of 0.022 .

\section{Discussion}

Our study shows the existence of a social differentiation in terms of patient satisfaction regarding the medical information delivered by caregivers: socially deprived patients were less satisfied with the information delivered about their health status and treatment, and with the quality and the duration of their interactions with the physician. The patients' perceived involvement in medical decision-making seemed to play an important role in this social differentiation. Indeed, in our model, this latent factor was strongly and positively linked to the patients' satisfaction regarding medical information and was negatively linked to social deprivation. The quality and duration of the patients' interactions with their physicians and nurses also seemed to have a significant impact on their satisfaction.

In previous studies, gender, age, ethnic origin, educational and marital status have all been identified as determinants of 
patient satisfaction $[6,26,27]$, but the analyses of the impact of socioeconomic indicators were frequently inconclusive [6, $28]$. A few studies, although, suggested that a lower socioeconomic status could be correlated with a lower satisfaction. Twenty years ago, Davis et al. [29] found that the rates of dissatisfaction with managed care were the highest among lower socioeconomic statuses and minorities. A more recent study in the field of primary care concluded that the most important element of patients' overall satisfaction was the quality of the communication with their doctor and also observed that patients living in deprived areas were usually less satisfied [30]. Our results also suggest a central role of the physician. Indeed, socially deprived patients were less satisfied with the information exclusively delivered by the physicians, i.e. information regarding their health status and their treatment.

Willems et al. [12] explored the influence of patients' socioeconomic status and physician-patient communications and showed that patients from lower social classes were given significantly less information and directions. Our study supports these results, showing that patients' perceived satisfaction about the quality and the duration of physician interactions is significantly lower for deprived patients. Vulnerable patients seem to be doubly penalized: patients from lower social class communicate less actively and express fewer information needs, while concurrently, physicians seem to display more patient-centered communication with patients who are perceived as better communicators, and who expressed positive affect [3]. Moreover, several authors demonstrated that positive physician-patient interaction and communication seem to be facilitated when physicians see themselves as close to their patients in terms of socioeconomic identities and values $[3,7,14]$.

Finally, our findings indicate that socially deprived patients feel more frequently that they are not involved in medical decision-making. Krupat et al. [31] showed that patients with a lower educational level were less interested in participating in medical decision-making. It seems therefore that vulnerable patients are caught in a vicious circle affecting their healthcare trajectory: first of all because of their more passive communication style and secondly because of the caregivers' misperception of their expectations and need for information. This social differentiation in medical information access leads in turn to health inequalities by omission, in the particular context of hospitalizations.

Our study was based on an original design: contrary to most published papers focusing on primary care and on doctor-patient interaction, we explored secondary care and included the perception of nurses. However, this study had a number of limitations. First, we obtained data on $76 \%$ of the eligible patients: this raises the possibility of a selection bias. An additional limitation is the potential for measurement error, especially a social desirability bias due to face-to-face interviews with patients and caregivers. Moreover, the EPICES score used for the detection of deprivation has been developed for primary care and not for hospitalized patients. However, it has already been used for inpatients in previous studies [24, 32] and seems to fit in the context of hospitalization, even if some of the items have to be adapted for elderly patients (for example, the item 'Have you done any sport activities in the last 12 months'). The EPICES score also has the advantage of being adapted to the French context and of taking into account multiple dimensions of the socioeconomic conditions-including psychological, social, and economic aspects. The binary categorization of social deprivation may also be questioned and some refinements on the notion of social deprivation should probably be considered in order to elaborate interventions that are adapted to every scenario. Lastly, our study does not provide any direct evidence about the quality of care and it is unclear whether these differences in perceptions are associated with real differences in care or outcomes. However, several studies illustrated the strong relationship between low socioeconomic status and poorer health outcomes $[16,19]$.

\section{Conclusion}

Social inequalities in health are a major challenge in modern healthcare systems. To tackle these inequalities, drivers of successful interventions targeted towards vulnerable groups of patients need to be identified.

First, the detection of social vulnerability during care provision could be improved and/or integrated in the hospital's computerized information systems. Assessing patients' objective and subjective state of deprivation, as well as their social history and life course perspective should also become common practice, assuming that it would change the way caregivers relate to their patient.

Furthermore, patients' participation should be promoted in order to facilitate their empowerment. Golin et al. [33] suggested that vulnerable patients whose physicians facilitate participation in clinical decision-making were more satisfied with their care. Greater patient participation has the potential to improve adherence to treatment and health outcomes.

A last approach would be to offer training in communication techniques to physicians and other healthcare professionals. Medical training often ignores cultural, social and psychological aspects of care. Recently, however, several initial or on-the-job courses for practitioners focusing on these relational aspects have been developed, in particular in the English-speaking world. This dimension is indeed central, and several studies have shown the beneficial effect of communication between patients and healthcare staff on the quality of care [34, 35]. 
In France, the lack of studies exploring patients' involvement in medical decisions and the role played by social differentiation creates a critical knowledge gap that should be filled. Further research on these subjects is therefore needed in order to improve patients' satisfaction and reduce health social inequalities.

\section{Acknowledgments}

The authors thank the Conservatoire national des Arts et Métiers (CNAM) who made language editing of the manuscript.

The project received funding from the 'Institut de recherche en santé publique' (IReSP) in 2009 (Project number: PROG/09/33).

\section{Availability of data and materials}

Dataset in spreadsheet format is available upon request to the first author.

\section{Authors' contributions}

LM and AP designed the study. AP collected the data. EA, FB and BL analyzed the data. $L M$ wrote the first draft of the manuscript. BL and FB revised the draft. All authors read and approved the final manuscript.

\section{Ethics approval and consent to participate}

The research protocol has obtained approval from the "Groupe nantais d'éthique dans le domaine de la santé (Gneds)". All eligible inpatients, physicians and nurses were invited to participate by the interviewer and gave their verbal informed consent before the interview, and the data were anonymized before recording. According to the L1121-1 and R1121-2 articles of the French code of public health, written consent and IRB approval are not necessary for non-interventional research.

\section{Competing interests}

The authors declare that they have no competing interests.

\section{Publisher's Note}

Springer Nature remains neutral with regard to jurisdictional claims in published maps and institutional affiliations.

\section{Author details}

${ }^{1}$ Public Health Department, University Hospital, Saint-Jacques Hospital, 85, rue Saint-Jacques, 44093 Nantes Cedex, France. ' UMR 1246 INSERM SPHERE "MethodS in Patients-centered outcomes and HEalth ResEarch", University of Nantes, Bd Benoni-Goullin, 44200 Nantes, France. ${ }^{3}$ BIOEPAR, INRA, Oniris, 44307 Nantes, France.

Received: 18 September 2016 Accepted: 14 November 2017 Published online: 23 November 2017

\section{References}

1. Street RL Jr. How clinician-patient communication contributes to health improvement: modeling pathways from talk to outcome. Patient Educ Couns. 2013;92(3):286-91.

2. Stewart MA. Effective physician-patient communication and health outcomes: a review. CMAJ. 1995;152(9):1423-33.

3. Street RL Jr, Gordon H, Haidet P. Physicians' communication and perceptions of patients: is it how they look, how they talk, or is it just the doctor? Soc Sci Med. 2007:65(3):586-98.

4. Marcinowicz L, Chlabicz S, Grebowski R. Patient satisfaction with healthcare provided by family doctors: primary dimensions and an attempt at typology. BMC Health Serv Res. 2009;9:63.

5. Schoenfelder T, Klewer J, Kugler J. Determinants of patient satisfaction: a study among 39 hospitals in an in-patient setting in Germany. Int J Qual Health Care. 2011;23(5):503-9.

6. Crow R, Gage H, Hampson S, Hart J, Kimber A, Storey L, Thomas H. The measurement of satisfaction with healthcare: implications for practice from a systematic review of the literature. Health Technol Assess. 2002;6(32):1-244.

7. Street RL Jr, O'Malley KJ, Cooper LA, Haidet P. Understanding concordance in patient-physician relationships: personal and ethnic dimensions of shared identity. Ann Fam Med. 2008;6(3):198-205.
8. Legare F, Turcotte S, Stacey D, Ratte S, Kryworuchko J, Graham ID Patients' perceptions of sharing in decisions: a systematic review of interventions to enhance shared decision making in routine clinical practice. Patient. 2012:5(1):1-19.

9. Branda ME, LeBlanc A, Shah ND, Tiedje K, Ruud K, Van Houten H, Pencille L, Kurland M, Yawn B, Montori VM. Shared decision making for patients with type 2 diabetes: a randomized trial in primary care. BMC Health Serv Res. 2013;13:301.

10. van Ryn M, Burgess D, Malat J, Griffin J. Physicians' perceptions of patients' social and behavioral characteristics and race disparities in treatment recommendations for men with coronary artery disease. Am J Public Health 2006;96(2):351-7.

11. Martin E, Russell D, Goodwin S, Chapman R, North M, Sheridan P. Why patients consult and what happens when they do. BMJ. 1991;303:289-92.

12. Willems S, De Maesschalck S, Deveugele M, Derese A, De Maeseneer J. Socio-economic status of the patient and doctor-patient communication: does it make a difference? Patient Educ Couns. 2005:56(2):139-46.

13. Kelly-Irving M, Delpierre C, Schieber AC, Lepage B, Rolland C, Afrite A, Pascal J, Cases $C$, Lombrail P, Lang T. Do general practitioners overestimate the health of their patients with lower education? Soc Sci Med. 2011;73(9):1416-21.

14. Schieber AC, Kelly-Irving M, Delpierre C, Lepage B, Bensafi A, Afrite A, Pascal J, Cases C, Lombrail P, Lang T. Is perceived social distance between the patient and the general practitioner related to their disagreement on patient's health status? Patient Educ Couns. 2013;91(1):97-104.

15. Gordon HS, Street RL Jr, Kelly PA, Souchek J, Wray NP. Physician-patient communication following invasive procedures: an analysis of postangiogram consultations. Soc Sci Med. 2005;61(5):1015-25.

16. Greenberg CC, Schneider EC, Lipsitz SR, Ko CY, Malin JL, Epstein AM, Weeks JC, Kahn KL. Do variations in provider discussions explain socioeconomic disparities in postmastectomy breast reconstruction? J Am Coll Surg. 2008;206(4):605-15.

17. Bensing JM, Tromp F, van Dulmen S, van den Brink-Muinen A, Verheul W, Schellevis FG. Shifts in doctor-patient communication between 1986 and 2002: a study of videotaped general practice consultations with hypertension patients. BMC Fam Pract. 2006;7:62.

18. Bao Y, Fox SA, Escarce JJ. Socioeconomic and racial/ethnic differences in the discussion of cancer screening: "between-" versus "within-" physician differences. Health Serv Res. 2007:42(3 Pt 1):950-70.

19. Ayanian JZ, Cleary PD, Keogh JH, Noonan SJ, David-Kasdan JA, Epstein AM. Physicians' beliefs about racial differences in referral for renal transplantation. Am J Kidney Dis. 2004;43(2):350-7.

20. Moret L, Rochedreux A, Chevalier S, Lombrail P, Gasquet I. Medical information delivered to patients: discrepancies concerning roles as perceived by physicians and nurses set against patient satisfaction. Patient Educ Couns. 2008;70(1):94-101.

21. Sass C, Gueguen R, Moulin JJ, Abric L, Dauphinot V, Dupre C, Giordanella JP, Girard F, Guenot C, Labbe E, et al. Comparison of the individual deprivation index of the French Health Examination Centres and the administrative definition of deprivation. Sante Publique. 2006;18(4):513-22.

22. Labbe E, Blanquet M, Gerbaud L, Poirier G, Sass C, Vendittelli F, Moulin JJ. A new reliable index to measure individual deprivation: the EPICES score. Eur J Pub Health. 2015;25(4):604-9.

23. Cosson E, Bihan H, Reach G, Vittaz L, Carbillon L, Valensi P. Psychosocial deprivation in women with gestational diabetes mellitus is associated with poor fetomaternal prognoses: an observational study. BM Open. 2015:5:e007120

24. Bihan H, Laurent S, Sass C, Nguyen G, Huot C, Moulin JJ, Guegen R, Le Toumelin P, Le Clesiau H, La Rosa E, et al. Association among individual deprivation, glycemic control, and diabetes complications: the EPICES score. Diabetes Care. 2005;28(11):2680-5.

25. Rosseel Y. Lavaan: an R package for structural equation modeling. J Stat Softw. 2012;48:1-36.

26. Nguyen Thi PL, Briancon S, Empereur F, Guillemin F. Factors determining inpatient satisfaction with care. Soc Sci Med. 2002;54(4):493-504.

27. Quintana JM, Gonzalez N, Bilbao A, Aizpuru F, Escobar A, Esteban C, SanSebastian JA, de-la- Sierra E, Thompson A. Predictors of patient satisfaction with hospital health care. BMC Health Serv Res. 2006:6:102.

28. Hall JA, Dornan MC. Patient sociodemographic characteristics as predictors of satisfaction with medical care: a meta-analysis. Soc Sci Med. 1990;30(7):811-8.

29. Davis K, Collins KS, Schoen C, Morris C. Choice matters: enrollees' views of their health plans. Health Aff (Millwood). 1995;14(2):99-112. 
30. Paddison CA, Abel GA, Roland MO, Elliott MN, Lyratzopoulos G, Campbell JL. Drivers of overall satisfaction with primary care: evidence from the English General Practice Patient Survey. Health Expect. 2015;18(5):1081-92.

31. Krupat E, Bell RA, Kravitz RL, Thom D, Azari R. When physicians and patients think alike: patient-centered beliefs and their impact on satisfaction and trust. J Fam Pract. 2001:50(12):1057-62.

32. Nahon S, Lahmek P, Macaigne G, Faurel JP, Sass C, Howaizi M, Fleury A, Baju A, Locher C, Barjonet G, et al. Socioeconomic deprivation does not influence the severity of Crohn's disease: results of a prospective multicenter study. Inflamm Bowel Dis. 2009;15(4):594-8.

33. Golin C, DiMatteo MR, Duan N, Leake B, Gelberg L. Impoverished diabetic patients whose doctors facilitate their participation in medical decision making are more satisfied with their care. J Gen Intern Med. 2002;17(11):857-66.

34. Davis S, Kristjanson LJ, Blight J. Communicating with families of patients in an acute hospital with advanced cancer: problems and strategies identified by nurses. Cancer Nurs. 2003:26(5):337-45.

35. Teutsch C. Patient-doctor communication. Med Clin North Am. 2003; 87(5):1115-45.

\section{Submit your next manuscript to BioMed Central} and we will help you at every step:

- We accept pre-submission inquiries

- Our selector tool helps you to find the most relevant journal

- We provide round the clock customer support

- Convenient online submission

- Thorough peer review

- Inclusion in PubMed and all major indexing services

- Maximum visibility for your research

Submit your manuscript at www.biomedcentral.com/submit 